\title{
Parameter estimation for propagation along random rough surface by using line of sight data
}

\author{
Kazunori Uchida ${ }^{\mathrm{a}, *}$, Masafumi Takematsu ${ }^{\mathrm{b}}$, Jun-Hyuck Lee ${ }^{\mathrm{b}}$ and Junichi Honda ${ }^{\mathrm{c}}$ \\ ${ }^{\mathrm{a}}$ Department of Information and Communication Engineering, Fukuoka Institute of Technology, \\ Higashi-Ku, Fukuoka, Japan \\ ${ }^{\mathrm{b}}$ Graduate School of Engineering, Fukuoka Institute of Technology, Higashi-Ku, Fukuoka, Japan \\ ${ }^{c}$ Surveillance and Communications Department, Electronic Navigation Research Institute, Tokyo, \\ Japan
}

\begin{abstract}
This paper is concerned with a numerical procedure to estimate two parameters, amplitude modification factor $\alpha$ and propagation order of distance $\beta$, for electromagnetic (EM) propagation in complicated natural environments such as random rough surface (RRS). These two parameters are key parameters when we simulate field distributions along various types of RRS based on 1-ray model. We assume that the former parameter $\alpha$ can be evaluated by the visual planar angles of illuminated lines in case of 1D RRS and by the visual solid angles of illuminated planes in case of 2D RRS. We also assume that the latter parameter $\beta$ can be estimated not only by base station (BS) antenna height, similar to Okumura-Hata model simulating EM propagation in urban and suburban areas, but also by mobile station (MS) antenna height. In order to demonstrate validity of the proposed parameter estimation, we compare the numerical field distributions obtained by the 1-ray model using estimated two parameters with those computed by discrete ray tracing method (DRTM) which is an effective EM field solver. It is shown that both numerical results are in good agreement.
\end{abstract}

Keywords: Propagation, random rough surface, 1-ray model, field estimation, discrete ray tracing method

\section{Introduction}

Recently, demands for wireless communications, such as cellular phones, wireless local area networks (LAN), ad hoc networks as well as sensor networks, have been rapidly increasing. Base stations (BS) and/or mobile stations (MS) of these systems are often located in complicated natural or artificial propagation environments, such as desert, hilly mountain, sea surface, urban or suburban area, and so forth. Consequently, it is important for wireless communication engineers to estimate propagation characteristics accurately in order to construct reliable wireless networks in these complicated propagation environments [1].

So far, we have introduced 1-ray and 2-ray models from which we can easily estimate electric field distributions in many complicated propagation environments by using two parameters, amplitude modification factor $\alpha$ and propagation order of distance $\beta[2,3]$. The ray models have been devised so that

\footnotetext{
${ }^{*}$ Corresponding author: Kazunori Uchida, Department of Information and Communication Engineering, Fukuoka Institute of Technology (FIT), 3-30-1 Wajiro-Higashi, Higashi-Ku, Fukuoka 811-0295, Japan. E-mail: k-uchida@ fit.ac.jp.
} 
we can obtain electric field intensities corresponding to the path loss provided by Okumura-Hata model for propagation in urban, suburban and open areas as listed in Appendix A. The Okumura-Hata model indicates that the propagation order of distance $\beta$ is dependent only on BS antenna height while the amplitude modification factor $\alpha$ is dependent on communication areas, BS and MS antenna heights, and operating frequency [5-7].

The ray models have also been successively applied to EM wave propagation along various types of random rough surfaces (RRS) [8-10]. The most interesting feature of the 1-ray model is that we can evaluated EM field distributions by choosing $\alpha$ and $\beta$ appropriately, and thus we can also evaluate communication distance easily [1,9]. The parameters $\alpha$ and $\beta$ seem to be closely related to the statistical quantities of RRS, that is, deviation of height $d v$ and correlation length $c l$, because many spectral functions for RRS are well described by $d v$ and $c l$ [11]. At the present stage, however, we have no explicit mathematical expressions which could adequately describe the relationship between $(\alpha, \beta)$ and $(d v, c l)[5,6]$. The propagation order of distance $\beta$ might be dependent on BS and MS antenna heights. The amplitude modification factor $\alpha$, on the other hand, might be strongly associated with the height deviation $d v$ and correlation length $c l$ of RRS. In this context, it is significant to propose a procedure to estimate $\alpha$ by introducing equivalent planar or solid angle of illuminated regions of RRS and $\beta$ by using the field matching factor $\gamma$ numerically $[5,6]$.

The main purpose of this paper is to check the accuracy of the proposed method by comparing present numerical results with those computed by discrete ray tracing method (DRTM) [1]. First, we review the 1-ray and 2-ray models which play an important role in computing electric field distributions in complicated propagation environments. Second, we discuss how to solve the propagation order of distance $\beta$ and we show that $\beta$ can be estimated in terms of field matching factor $\gamma$ having correlation with BS and MS antenna heights. Third, we propose an estimation procedure for $\alpha$ by using the equivalent angle corresponding to illuminated planar and solid angles for 1D and 2D RRSs, respectively. Finally, we propose tentative analytical expressions for estimating $\alpha$ and $\beta$, and we also show some numerical examples for electric field distributions to demonstrate validity of the proposed method.

\section{Field expressions}

Now we review the 1-ray and 2-ray models characterized by introducing amplitude modification factor $\alpha$ and propagation order of distance $\beta$. It should be noted that the models are simple but we can estimate field distributions in many complicated propagation environments, such as urban, suburban and rural areas as well as RRSs. In other words, we can always evaluate electric field distributions in any propagation environments with the appropriately estimated parameters $\alpha$ and $\beta$. It should also be emphasized that the 1-ray model enables us to estimate communication distance in such complicated propagation environments.

\subsection{1-ray and 2-ray models}

The results are summarized in far zone $(r>>\lambda)$ as follows [2,3]:

$$
\boldsymbol{E}_{\mathbf{1}}=10^{\alpha / 20} 10^{(\beta-1) \gamma / 20} r^{(1-\beta)} \boldsymbol{E}_{\boldsymbol{i}}, \quad \boldsymbol{E}_{\mathbf{2}}=10^{\alpha / 20} 10^{(\beta-2) \gamma / 20} r^{(2-\beta)} \boldsymbol{E}_{\boldsymbol{t}}
$$

The first and second equations in Eq. (1) correspond to the 1-ray and 2-ray models, respectively. 


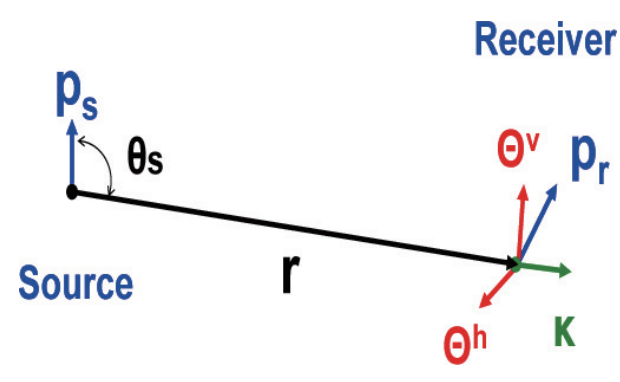

Fig. 1. Incident ray.

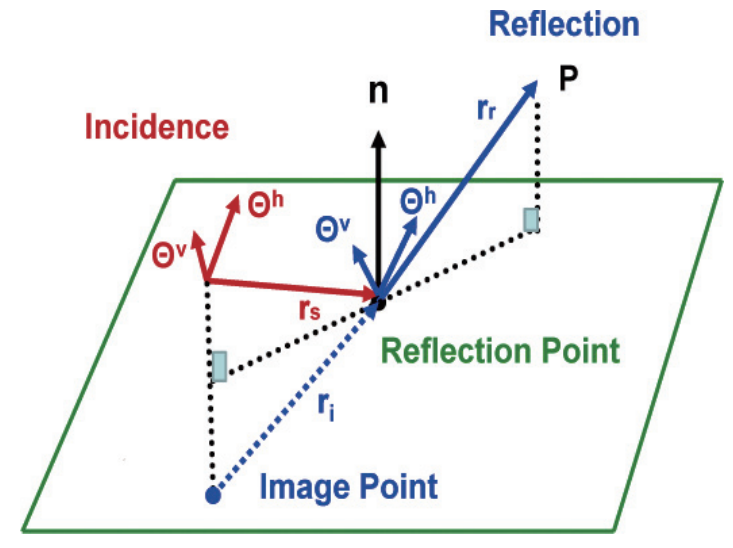

Fig. 2. Reflection ray.

The incident electric field $\boldsymbol{E}_{\boldsymbol{i}}$ in free space and the total electric field $\boldsymbol{E}_{\boldsymbol{t}}$ above a ground plane are employed for the first and second models, respectively. These two electric fields are expressed as follows $[12,13]$ :

$$
\boldsymbol{E}_{\boldsymbol{i}}=\sqrt{30 G_{s} P_{s}} \sin \theta \frac{e^{-j \kappa r}}{r} \Theta^{v}\left(\boldsymbol{r}, \boldsymbol{p}_{\boldsymbol{s}}\right), \boldsymbol{E}_{\boldsymbol{t}}=\boldsymbol{E}_{\boldsymbol{i}}+\sqrt{30 G_{s} P_{s}} \sin \theta_{0} \frac{e^{-j \kappa r_{0}}}{r_{0}} \boldsymbol{e}_{\boldsymbol{r}}
$$

where the time dependence $e^{j \omega t}$ is assumed. Moreover, $\omega=2 \pi f$ is an angular frequency with operating frequency $f, \kappa=2 \pi / \lambda$ is a wave number with wave length $\lambda$ in free space, and $r$ is a position vector from a source to a receiver as shown in Fig. 1. The electric field vector for a reflected wave from the ground plane is given by

$$
\left.\left.\boldsymbol{e}_{\boldsymbol{r}}=R^{v}\left(\theta_{i}\right)\left[\boldsymbol{\Theta}^{v}\left(\boldsymbol{r}_{\mathbf{1}}, \boldsymbol{p}_{\boldsymbol{s}}\right) \cdot \boldsymbol{\Theta}^{v}\left(\boldsymbol{r}_{\mathbf{1}}, \boldsymbol{n}\right)\right] \boldsymbol{\Theta}^{v}\left(\boldsymbol{r}_{\mathbf{2}}, \boldsymbol{n}\right)\right]+R^{h}\left(\theta_{i}\right)\left[\boldsymbol{\Theta}^{h}\left(\boldsymbol{r}_{\mathbf{1}}, \boldsymbol{p}_{\boldsymbol{s}}\right) \cdot \boldsymbol{\Theta}^{h}\left(\boldsymbol{r}_{\mathbf{1}}, \boldsymbol{n}\right)\right] \boldsymbol{\Theta}^{h}\left(\boldsymbol{r}_{\mathbf{2}}, \boldsymbol{n}\right)\right]
$$

where $r_{0}=r_{s}+r_{r}$ and $r_{s}$ is the distance from source to reflection point and $r_{r}$ is the distance from reflection point to receive point $P$ as shown in Fig. 2. The vertical and horizontal unit vectors used in the above relation are defined by

$$
\Theta^{v}\left(\boldsymbol{r}, \boldsymbol{p}_{\boldsymbol{s}}\right)=\frac{\left(\left(\boldsymbol{r} \times \boldsymbol{p}_{\boldsymbol{s}}\right) \times \boldsymbol{r}\right)}{\left|\left(\boldsymbol{r} \times \boldsymbol{p}_{\boldsymbol{s}}\right) \times \boldsymbol{r}\right|}, \Theta^{h}\left(\boldsymbol{r}, \boldsymbol{p}_{\boldsymbol{s}}\right)=\frac{\left(\boldsymbol{r} \times \boldsymbol{p}_{\boldsymbol{s}}\right)}{\left|\boldsymbol{r} \times \boldsymbol{p}_{\boldsymbol{s}}\right|} .
$$

Moreover, the field matching factor $\gamma[d B]$ used in Eq. (1) and the field matching length $\Gamma[m]$ is defined by use of Eq. (2) as follows:

$$
\gamma=20 \log _{10}(\Gamma)[d B], \quad \Gamma=\left(r\left|\boldsymbol{E}_{\boldsymbol{t}}\right| /\left|\boldsymbol{E}_{\boldsymbol{i}}\right|\right)[m] .
$$

When the distance $d$ between a base station (BS) and a mobile station (MB) is much larger than BS antenna height $h_{b}$ and MS antenna height $h_{m}$, the field matching factor can be well approximately in a simple expression as follows [7]:

$$
\gamma \simeq 20 \log _{10}\left(2 \kappa h_{b} h_{m}\right)[d B], \Gamma \simeq 2 \kappa h_{b} h_{m}[m],\left(d>>h_{b}, h_{m}\right) .
$$


The Fresnel reflection coefficients for the vertical and horizontal electric field components are given by $[12,13]$

$$
R^{h}\left(\theta_{i}\right)=\frac{\cos \theta_{i}-\sqrt{\epsilon_{c}-\sin ^{2} \theta_{i}}}{\cos \theta_{i}+\sqrt{\epsilon_{c}-\sin ^{2} \theta_{i}}}, \quad R^{v}\left(\theta_{i}\right)=\frac{\epsilon_{c} \cos \theta_{i}-\sqrt{\epsilon_{c}-\sin ^{2} \theta_{i}}}{\epsilon_{c} \cos \theta_{i}+\sqrt{\epsilon_{c}-\sin ^{2} \theta_{i}}}
$$

where $\epsilon_{c}=\epsilon_{r}-j \sigma / \omega \epsilon_{0}$ is the complex permittivity of the ground plane with dielectric constant $\epsilon_{r}$ and conductivity $\sigma$ together with permittivity $\epsilon_{0}$ in free space. In the subsequent numerical examples we have employed the parameters such that $\epsilon_{r}=5.0$ and $\sigma=0.0023[S / m]$ corresponding to a dry soil constituting the ground plane. Moreover, we have assumed that gains of source and receive antennas are $G_{s}=G_{r}=1.5$, directivity of source antenna is $\sin \theta$, directivity of receive antenna is $\sin \theta_{0}$ in Eq. (2), respectively, and $\theta_{i}$ in Eq. (7) is an incident angle of the incident ray at a reflection point. These angles are given by

$$
\sin \theta=\frac{\left|\boldsymbol{p}_{\boldsymbol{s}} \times \boldsymbol{r}\right|}{r}, \sin \theta_{0}=\frac{\left|\boldsymbol{p}_{\boldsymbol{s}} \times \boldsymbol{r}_{\boldsymbol{s}}\right|}{r_{s}}, \sin \theta_{i}=\frac{\left|\boldsymbol{n} \times \boldsymbol{r}_{\boldsymbol{s}}\right|}{r_{s}}
$$

where the distance $r=|\boldsymbol{r}|$ between source and receive antennas should be much larger than $\lambda$.

\subsection{Communication distance}

Since the direction vectors of the source and receive antennas are $\boldsymbol{p}_{s}$ and $\boldsymbol{p}_{\boldsymbol{r}}$ as shown in Fig. 1, received power of a small dipole antenna can be expressed as follows $[12,13]$ :

$$
P_{r}=\frac{\lambda^{2} G_{r}}{4 \pi} \cdot \frac{\left|\boldsymbol{E} \cdot \boldsymbol{p}_{\boldsymbol{r}}\right|^{2}}{Z_{0}}[\mathrm{~W}]
$$

where the intrinsic impedance in free space is given by $Z_{0} \simeq 120 \pi$ [ $\Omega$ ] [12]. From Eqs (1) and (2), on the other hand, the 1-ray model yields the following relation

$$
\left|\boldsymbol{E}_{\mathbf{1}}\right|=10^{\frac{\alpha}{20}} \cdot 10^{\frac{(\beta-1) \gamma}{20}} \cdot \frac{\sqrt{30 G_{s} P_{s}}}{r^{\beta}}
$$

where antenna orientation is assumed to be arranged so that the maximum received power may be obtained.

Let $E_{\min }$ be the minimum detectable electric field intensity and $D_{c}$ be the maximum communication distance. Then, Eq. (10) yields the following relation

$$
E_{\min }=10^{\frac{\alpha}{20}} \cdot 10^{\frac{(\beta-1) \gamma}{20}} \cdot \frac{\sqrt{30 G_{s} P_{s}}}{D_{c}^{\beta}} .
$$

Consequently, Eq. (11) leads to the maximum communication distance $D_{c}$ given by

$$
D_{c}=10^{\frac{\alpha}{20 \beta}} \times 10^{\frac{(\beta-1) \gamma}{20 \beta}} \times\left(B_{\min }\right)^{\frac{1}{2 \beta}}
$$

where the minimum parameter $B_{\min }$ is defined by using the minimum detectable electric field intensity $E_{\min }$ or the minimum detectable receiving power $P_{\min }$ as follows:

$$
B_{\min }=\frac{30 G_{s} P_{s}}{\left(E_{\min }\right)^{2}}=\frac{\lambda^{2} G_{r}}{4 \pi} \cdot \frac{30 G_{s} P_{s}}{Z_{0} P_{\min }} .
$$

It is evident from Eq. (9) that the minimum detectable power is related to the minimum detectable electric field intensity as follows:

$$
P_{\min }=\frac{\lambda^{2} G_{r}}{4 \pi} \cdot \frac{\left(E_{\min }\right)^{2}}{Z_{0}}
$$




\section{Path loss for propagation in urban areas}

Assuming ideal isotropic source and receive antennas with unit gain $G_{s}=G_{r}=1$ in accordance with the Okumura-Hata model [4], the received power in Eq. (9) can be rewritten as follows:

$$
P_{r}=\frac{\lambda^{2}}{4 \pi} \frac{|\boldsymbol{E}|^{2}}{Z_{0}} .
$$

This equation can be expressed in $[\mathrm{dB}]$ as follows:

$$
P_{r}[\mathrm{dBW}]=|\boldsymbol{E}|[\mathrm{dBV} / \mathrm{m}]-10 \log _{10} Z_{0}+10 \log _{10}\left(\lambda^{2} / 4 \pi\right) .
$$

Since the path loss $L_{p}$ expressed in $[\mathrm{dB}]$ is given by the difference between the transmitted power and the received power [4], the path loss can be described in [dB] as follows:

$$
L_{p}[\mathrm{~dB}]=P_{s}[\mathrm{dBW}]-P_{r}[\mathrm{dBW}]=P_{s}[\mathrm{dBW}]-|\boldsymbol{E}|[\mathrm{dBV} / \mathrm{m}]+10 \log _{10} Z_{0}-10 \log _{10}\left(\lambda^{2} / 4 \pi\right) .
$$

Equation (17) indicates that the received electric field can be obtained by the path loss $L_{p}$ as long as the input power $P_{s}$ is specified.

Assuming unit input power $P_{s}=1$ [W] for the 1-ray model in Eq. (1), the path loss in Eq. (17) is rewritten as follows:

$$
L_{p}[\mathrm{~dB}]=-\alpha-(\beta-1) \gamma+20 \beta \log _{10} r-10 \log _{10} 30+10 \log _{10} Z_{0}-10 \log _{10}\left(\lambda^{2} / 4 \pi\right) .
$$

Thus the 1-ray model ensures that a relation between $\alpha$ and $\beta$ can be explicitly described by Eq. (18), as far as $L_{p}$ is known experimentally or theoretically and Eq. (6) is satisfied. The path loss in urban, suburban or open area can be derived from the Okumura-Hata model as listed in Appendix A. One of the important features is that $\beta$ depends only on the source antenna height $h_{b}[\mathrm{~m}]$ as follows:

$$
\beta=\beta\left(h_{b}\right)=\left(44.9-6.55 \log _{10} h_{b}\right) / 20 .
$$

As a result, based on the Okumura-Hata model, it is evident that propagation order of distance $\beta$ is a monotonically decreasing function with respect to BS antenna height $h_{b}$ alone. Combining Eqs (18) and (19) leads to the explicit expression for $\alpha$ as follows:

$$
\alpha=-L_{p}[\mathrm{~dB}]-(\beta-1) \Gamma+20 \beta \log _{10} R+20 \log _{10} f_{c}+60 \beta+20 \log _{10}(4 \pi / 300)
$$

where some conversions in unit have been made in accordance with the Okumura-Hata model; that is, $f[\mathrm{~Hz}] \rightarrow f_{c}[\mathrm{MHz}]$ and $r[\mathrm{~m}] \rightarrow R[\mathrm{Km}]$. It should be noted that the above relation is restricted only to the urban, suburban and open areas. In other regions, however, we must interpolate $\alpha$ approximately [7].

\section{Estimation of $\alpha$ and $\beta$ for propagation along RRS}

The Okumura-Hata model model shows that the propagation order of distance $\beta$ can be evaluated by BS antenna height $h_{b}$ alone and the modification factor $\alpha$ can be obtained by the path loss in the urban, suburban and open areas. In this section we propose a procedure to estimate $\alpha$ and $\beta$ numerically in case of EM propagation along RRSs [5,6]. 


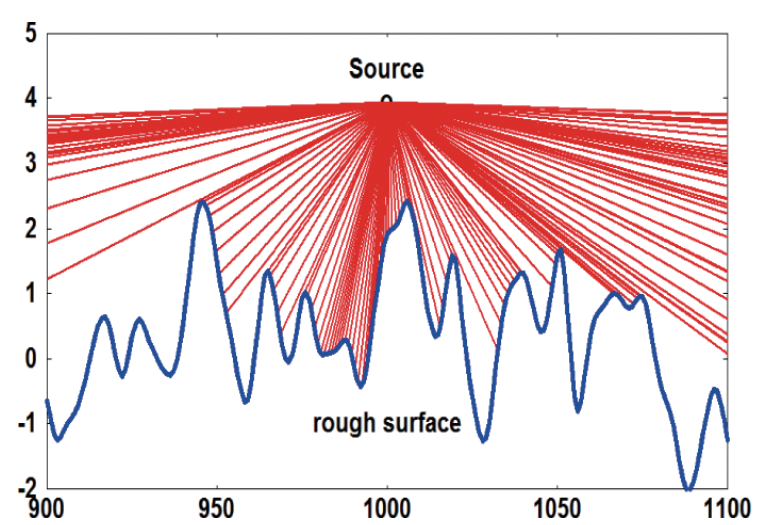

Fig. 3. Incident rays on 1D RRS.

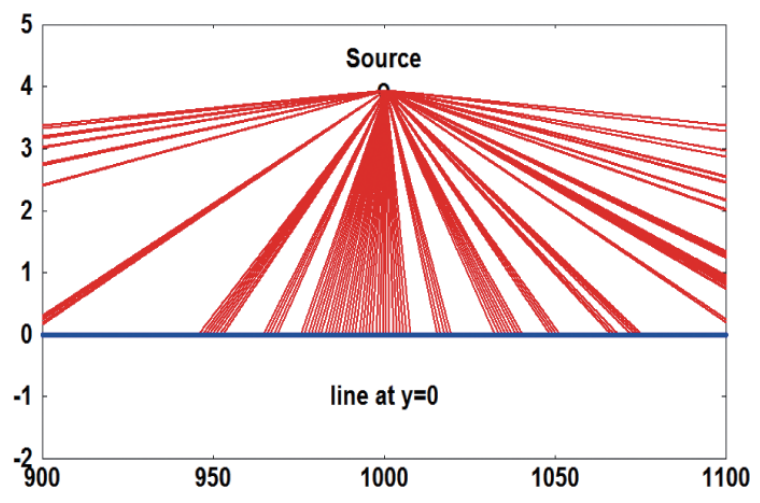

Fig. 4. Illuminated regions for 1D RRS.

Considering the EM propagation along a uniform ground plane with parameters $\alpha=0$ and $\beta=\beta_{0}=$ 1 in Eq. (1), Eq. (12) yields the following communication distance

$$
D_{c}=10^{\frac{\left(\beta_{0}-1\right) \gamma}{20 \beta_{0}}} \times\left(B_{\min }\right)^{\frac{1}{2 \beta_{0}}} .
$$

The above communication distance is increased for a large BS antenna height, since the field matching factor $\gamma$ is increased for large $h_{b}$ as shown in Eq. (6). When the BS antenna height is increased from $h_{b 0}$ to $h_{b}$, the propagation order of distance is changed from $\beta_{0}$ to $\beta$ and the field matching factor is varied from $\gamma_{0}$ to $\gamma$. Then, assuming that the analytical property of Eq. (21) with respect to $\beta$ is unchanged together with $\gamma$, we have the following relation

$$
10^{\frac{\left(\beta_{0}-1\right) \gamma}{20 \beta_{0}}} \times\left(B_{\min }\right)^{\frac{1}{2 \beta_{0}}} \simeq 10^{\frac{(\beta-1) \gamma_{0}}{20 \beta}} \times\left(B_{\min }\right)^{\frac{1}{2 \beta}}
$$

which can be solved for $\beta$ as follows:

$$
\beta=\beta(\gamma) \simeq \frac{\beta_{0}\left(\gamma_{0}-10 \log _{10} B_{\min }\right)}{\beta_{0} \gamma_{0}-\left(\beta_{0}-1\right) \gamma-10 \log _{10} B_{\min }} .
$$

It has been demonstrated that $\beta$ computed by Eq. (23) is fairly in good agreement with the OkumuraHata model [5]. Anyway the most interesting feature is that $\beta$ is given by a function of $\gamma$ or $\beta(\gamma)$ which has a monotonically decreasing property, in contrast to the Okumura-Hata model where $\beta$ is given by a function of $h_{b}$ or $\beta\left(h_{b}\right)$ as shown in Eq. (19). As is evident from Eq. (6), it is concluded that the propagation oder of distance is dependent not only BS antenna height but also MS antenna height as well as operating frequency.

We propose a procedure to estimate $\alpha$ by considering the effective planar angles for illuminated segments. First, we approximate propagation characteristic by taking account of the incident rays as shown in Fig. 3. Then we project the illuminated segments on the straight line of at $y=0$ as shown in Fig. 4, and we sum up the planar angles corresponding to the illuminated segments. We denote the total angle as equivalent angle $\theta_{e}$ of incidence. Next, we assume that the equivalent angle concentrates near the nadir of the source antenna as shown in Fig. 5 where $R$ is a maximum communication distance and $R_{e}$ is an equivalent communication distance [5]. The equivalent communication distance is defined by

$$
R_{e}=2 h_{b} \tan \left(\theta_{e} / 2\right) .
$$




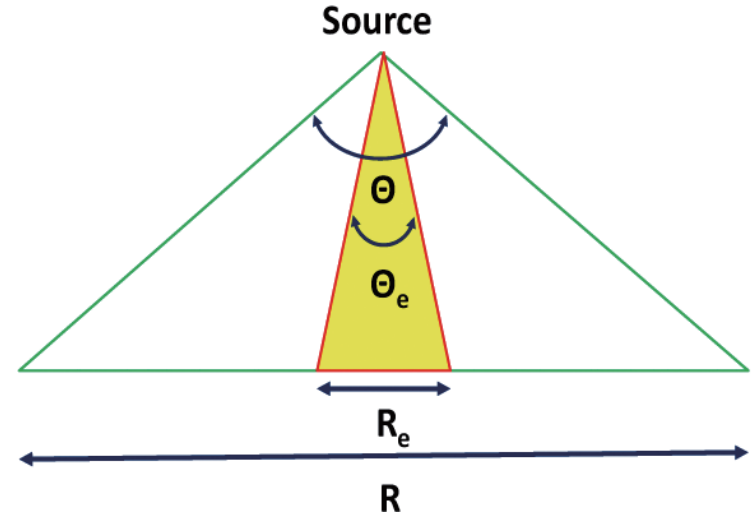

Fig. 5. Equivalent angle for 1D RRS.

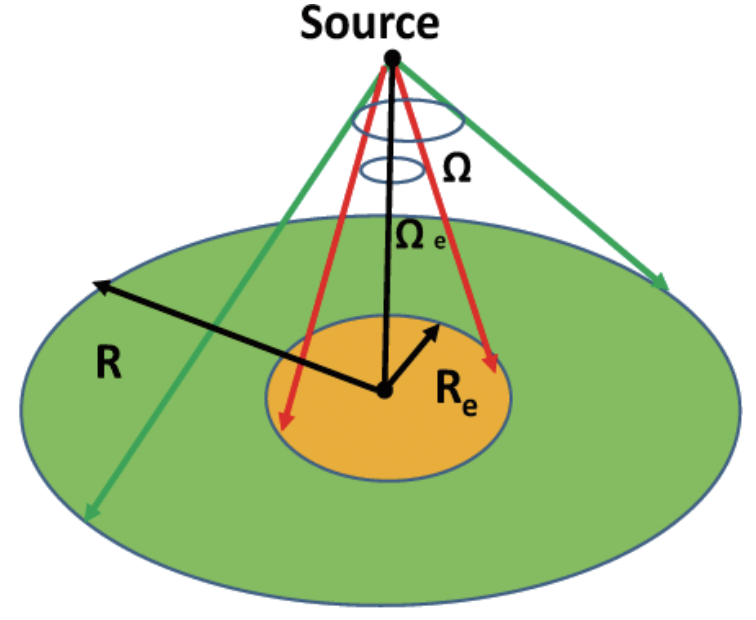

Fig. 6. Equivalent angle for 2D RRS.

Finally we assume that $\alpha$ might be given by a function of the equivalent length as follows:

$$
\alpha=K \log _{10}\left(R_{e} / R\right)
$$

where $K$ is a constant which might be in proportion to wave number $\kappa$.

In case of 2D RRS, extension from the planar angle to the solid angle [14] is enough to obtain the equivalent angle $\theta_{e}$ by summing up each solid angle corresponding to every illuminated triangular plate projected on the average plane at $z=0$. The analytical relation between a planar triangle and corresponding solid angle is shown in Appendix B.

The total solid angle $\Omega$ and the planar angle $\theta$ are related by

$$
\Omega=2 \pi(1-\cos \theta)
$$

and the illuminated equivalent angle for 2D RRS is given by

$$
\theta_{e}=\cos ^{-1}\left(1-\Omega_{e} / 2 \pi\right) .
$$

Thus, the amplitude modification factor $\alpha$ in case of 2D RRS can be estimated by use of Eqs (25) and (27). The physics of this theory is that electric field distributions along RRSs are mainly influenced by the optical situation whether receiving point is in line-of-sight (LOS) or in non-line-of-sight (NLOS).

\section{DRTM}

We calculate EM field distributions along RRS by using DRTM in order to check the accuracy of the 1-ray model which includes two parameters $\alpha$ and $\beta$ estimated by the numerical procedure proposed in this paper. RRS generation, on the other hand, is performed by the convolution method which is prescribed by two parameters, height deviation $d v$ and correlation length $c l$, together with type of rough surface spectrum [11]. In this paper we deal with only the Gaussian type of spectrum.

The first step of DRTM is to discretize RRS profile in terms of straight lines for 1D case and rectangular plates for 2D case, respectively. Thus, the RRS profile can be approximated by piece wise linear 


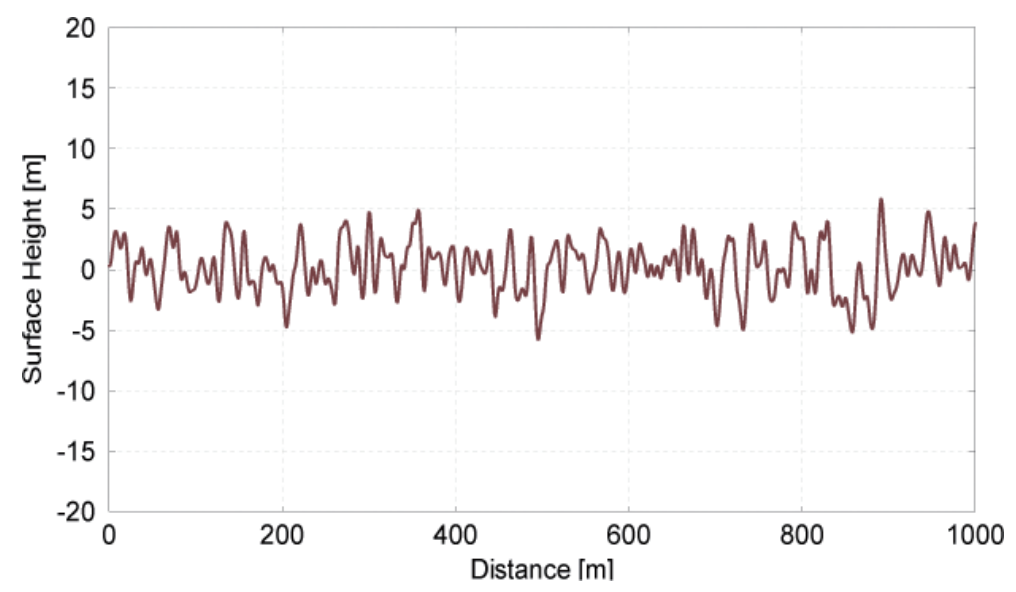

Fig. 7. An example of 1D RRS.

lines or piece wise planar plates, and this discretization should be performed so that computer memory together with numerical errors could be as small as possible. The second step is to discretize the procedure for searching rays traveling along RRS. To do this, we assume that arbitrary two lines (1D) or plates (2D) composing a RRS profile are in LOS if a representative point of one line or plate is in LOS with that of another line or plate, and otherwise, they are in NLOS. This assumption enables us to simplify ray searching drastically, resulting in saving much computation time [1]. It is worth noting that this algorithm can be modified to achieve more accurate rays.

Once necessary rays are searched for BS and MS antennas arbitrarily located along a generated RRS, we can evaluate EM fields in terms of searched ray data together with RRS data; the ray data are reflection and diffraction points composed of representative points of discretized lines or plates, and the RRS data are position and normal vectors of lines or plates of a discretized RRS profile. Detailed discussions are omitted here, but the electric field $\boldsymbol{E}$ at a receive point is formally expressed in the following dyadic and vector form [1]:

$$
\boldsymbol{E}=\sum_{n=1}^{N}\left[\prod_{m=1}^{m=M_{n}^{r}}\left(\boldsymbol{R}_{\boldsymbol{n m}}\right) \cdot \prod_{k=1}^{k=M_{n}^{d}}\left(\boldsymbol{D}_{\boldsymbol{n} \boldsymbol{k}}\right) \cdot \boldsymbol{E}_{\mathbf{0}}\right] \frac{e^{-j \kappa r_{n}}}{r_{n}}
$$

where $\boldsymbol{R}_{\boldsymbol{n} \boldsymbol{m}}$ is the dyadic for reflection at the $m$-th reflection point of the $n$-ray, $\boldsymbol{D}_{\boldsymbol{n} \boldsymbol{k}}$ is the dyadic dor diffraction at the $k$-th diffraction point of the $n$-ray, and $\boldsymbol{E}_{\mathbf{0}}$ is the electric field of the $n$-th ray at the first reflection or diffraction point. Moreover, $r_{n}$ is the distance of the $n$-th ray from BS antenna to MS antenna, $N$ is the total number of rays considered, $M_{n}^{d}$ is the number of times of source diffractions of the $n$-th ray, and $M_{n}^{r}$ is the number of times of image diffractions of the $n$-th ray.

\section{Numerical examples}

Figure 7 shows a RRS example where parameters are chosen as $d v=2[\mathrm{~m}]$ and $c l=5[\mathrm{~m}]$. The BS antenna is located at $x=0$ with height $h_{b}$ above RRS, and the MS antenna height is 1 [m] above RRS. In the following numerical examples, operating frequency is selected as $f=300[\mathrm{MHz}]$ and material constants of RRS are chosen as $\epsilon_{r}=5$ and $\sigma=0.0023[\mathrm{~S} / \mathrm{m}]$. 


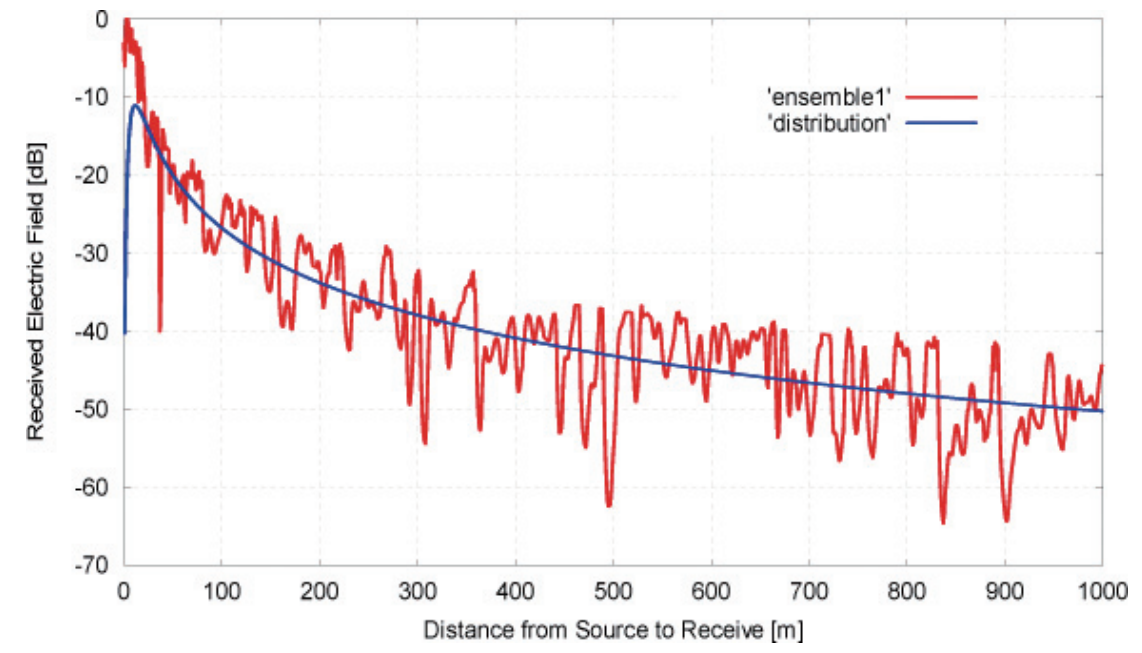

Fig. 8. Estimated field compared with DRTM.

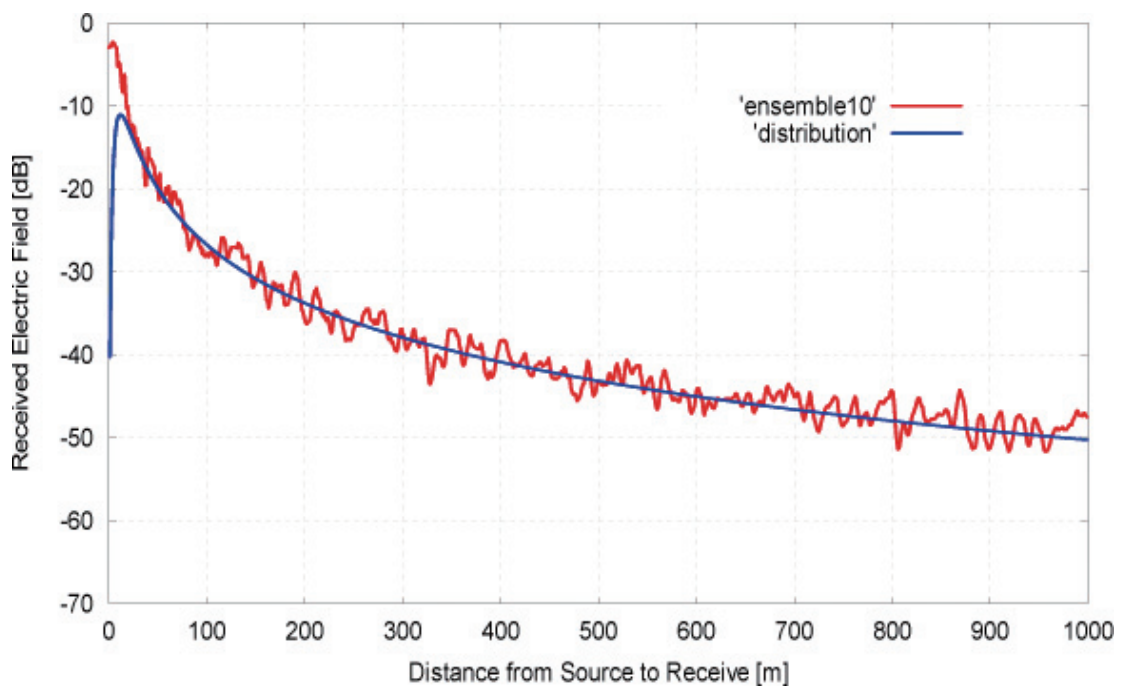

Fig. 9. Estimated field compared with DRTM.

Figure 8 shows electric field distribution computed by DRTM for one generated RRS with $d v=2$ [m], $c l=5[\mathrm{~m}]$ and $h_{b}=10[\mathrm{~m}]$ in comparison with the 1-ray model in Eq. (1) where $\alpha$ and $\beta$ are tentatively estimated by the following relations:

$$
\alpha=K \log _{10}\left(R_{e} / R\right), \quad \beta=\left(2 w^{2}+\gamma^{2}\right) /\left(w^{2}+\gamma^{2}\right)
$$

where $K=5$ and $w=20$ have been assumed. This parameter setting is employed in all the numerical examples listed hereafter. It should be noted that the second relation in Eq. (29) is arranged so that $\beta \rightarrow 1$ for large antenna heights, that is, for small $\gamma$ and $\beta \rightarrow 2$ for small antenna heights, that is, for small $\gamma$. Fig. 9 shows ensemble averaged electric field distribution computed by DRTM for ten generated RRSs with $d v=2[\mathrm{~m}], c l=5[\mathrm{~m}]$ and $h_{b}=10[\mathrm{~m}]$ in comparison with the 1-ray model using $\alpha$ and $\beta$ 


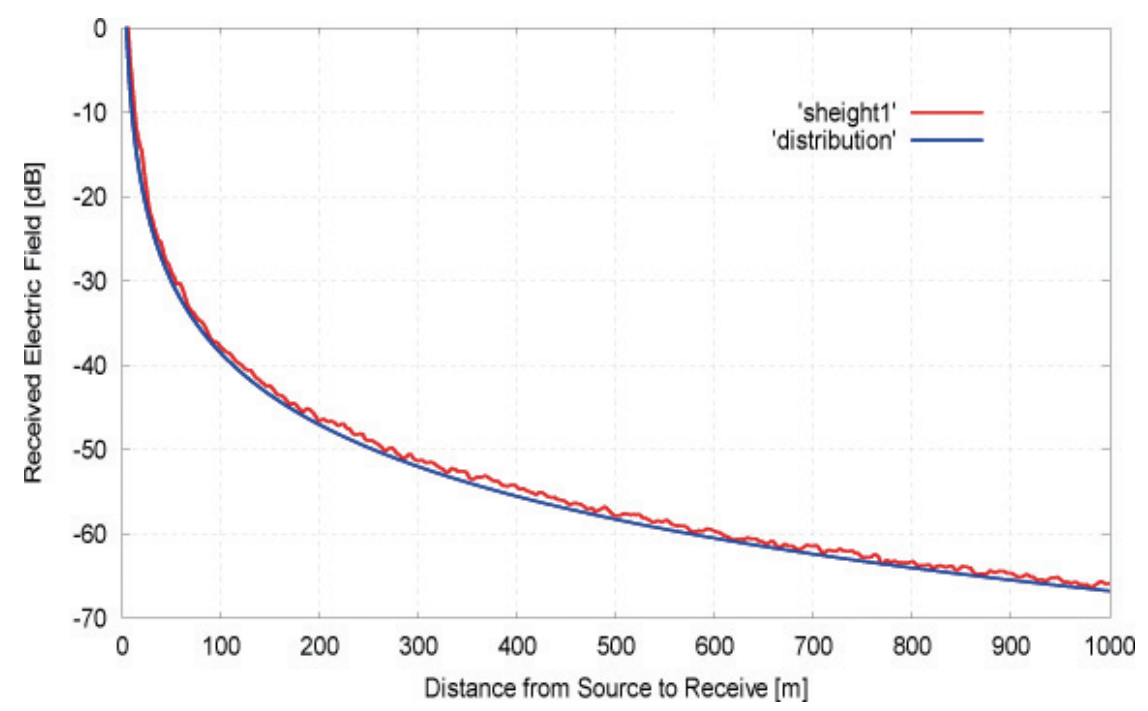

Fig. 10. Estimated field compared with DRTM.

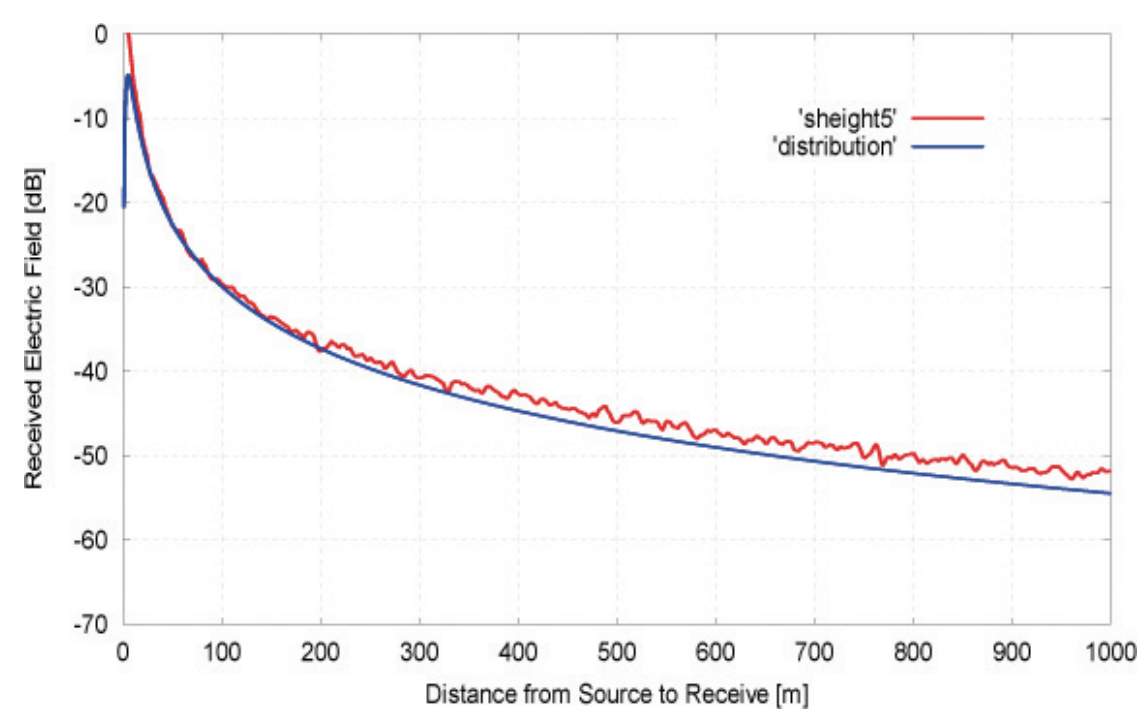

Fig. 11. Estimated field compared with DRTM.

estimated by Eq. (29). It is well demonstrated that the estimated field distributions based on the 1-ray model employing the proposed parameters are in good agreement with the ensemble average of field distributions computed by DRTM even if the number of RRS samples is only ten.

Figures 10, 11 and 12 show electric field distributions of 100 ensemble average of DRTM results in comparison with the estimated fields based on the proposed 1-ray model using parameters estimated by Eq. (29) for $h_{b}=1[\mathrm{~m}], 5[\mathrm{~m}]$ and $10[\mathrm{~m}]$, respectively. Other parameters are chosen as the same as Figs 8 and 9. It is shown that the numerical results of the proposed field estimation procedure provide a good accuracy in the NLOS-dominated case $\left(h_{b}=1[\mathrm{~m}]\right)$ as well as in the LOS-dominated case $\left(h_{b}=10[\mathrm{~m}]\right)$, and also a fairly good accuracy in the grazing-angle case $\left(h_{b}=5[\mathrm{~m}]\right)$. 


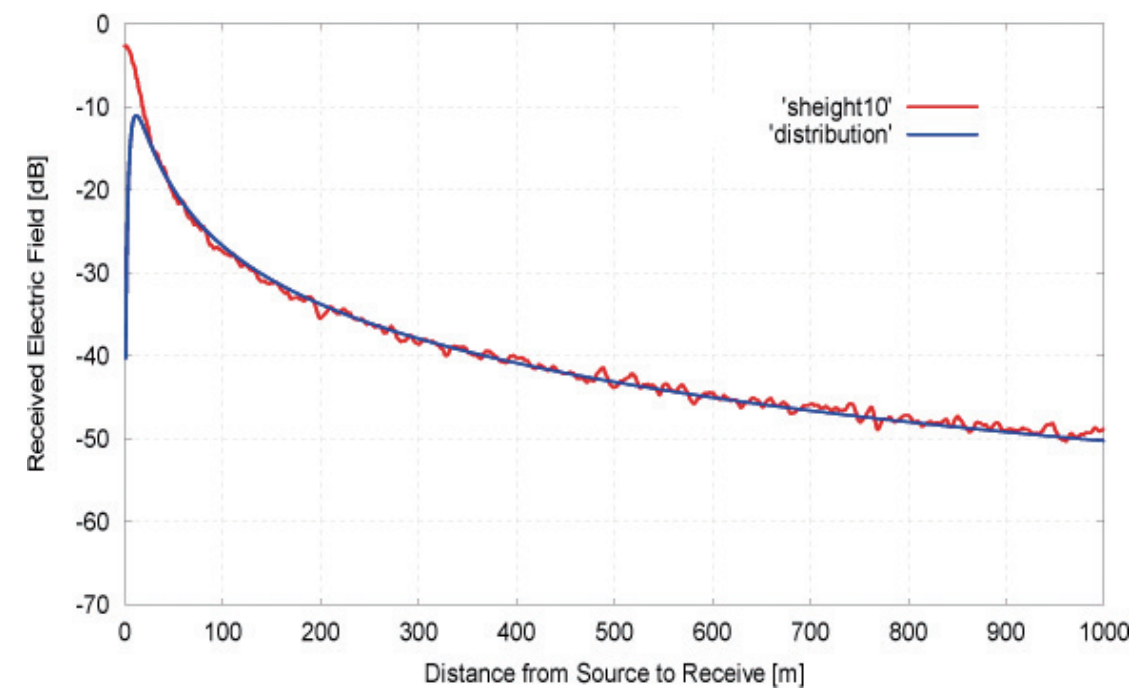

Fig. 12. Estimated field compared with DRTM.

\section{Conclusion}

First, we have reviewed the 1-ray and 2-ray models in order to estimate approximately propagation characteristics in urban, suburban and open areas as well as along RRS. Second, we have discussed two propagation parameters, amplitude modification factor $\alpha$ and propagation order of distance $\beta$, which play an important role for estimating communication distance in complicated propagation environments. Third, we have proposed an algorithm to estimate $\alpha$ by computing equivalent angle or length related to the illuminated portion of RRS, and we have also introduced a procedure to estimate $\beta$ in terms of field matching factor $\gamma$ which is used by in the 1-ray model. Finally, we compared the field distributions obtained by the proposed method with the ensemble averaged DRTM solutions.

It has been demonstrated that the proposed methods are useful for propagation estimation in complicated natural or artificial environments, because the proposed method can be applied effectively not only in LOS-region but also NLOS-region. It is needed to consider other situations with different frequency and other RRS parameters. This deserves as a future investigation.

\section{Acknowledgments}

The work was supported in part by a Grand-in Aid for Scientific Research (C) (24560487) from Japan Society for the Promotion of Science.

\section{Okumura-Hata model}

Empirical equations for the path loss in urban, suburban and open areas are summarized as follows [4]:

- Urban Area:

$$
L_{p}[d B]=69.55+26.16 \log _{10} f_{c}-13.82 \log _{10} h_{b}-a\left(h_{m}\right)+\left(44.9-6.55 \log _{10} h_{b}\right) \log _{10} R
$$


where modifying factor for the height of the mobile antenna $h_{m}$ is given by

$$
\begin{array}{rlrl}
a\left(h_{m}\right) & =\left(1.1 \log _{10} f_{c}-0.7\right) h_{m}-\left(1.56 \log _{10} f_{c}-0.8\right) & & (\text { in medium - small city }) \\
a\left(h_{m}\right) & =8.29\left(\log _{10} 1.54 h_{m}\right)^{2}-1.1 ; f_{c} \leqslant 200[\mathrm{MHz}] \quad & (\text { in large city }) \\
& =3.2\left(\log _{10} 11.75 h_{m}\right)^{2}-4.97 ; f_{c} \geqslant 400[\mathrm{MHz}] \quad \text { (in large city) }
\end{array}
$$

- Suburban Area:

$$
L_{p s}[d B]=L_{p}\{\text { Urban area }\}-2\left\{\log _{10}\left(f_{c} / 28\right)\right\}^{2}-5.4
$$

- Open Area:

$$
L_{p o}[d B]=L_{p}\{\text { Urban area }\}-4.78\left(\log _{10} f_{c}\right)^{2}+18.33 \log _{10} f_{c}-40.94
$$

The carrier frequency $f_{c}[\mathrm{MHz}]$ is confined from $150[\mathrm{MHz}]$ to $1500[\mathrm{MHz}]$, the antenna height $h_{b}[\mathrm{~m}]$ of BSs is limited from $30[\mathrm{~m}]$ to $200[\mathrm{~m}]$, the antenna height $h_{m}[\mathrm{~m}]$ of MSs is ranged from $1[\mathrm{~m}]$ to $10[\mathrm{~m}]$, and the communication distance $R[\mathrm{Km}]$ is confined from $1[\mathrm{Km}]$ to $20[\mathrm{Km}]$.

\section{B. Solid angle}

Based on the spherical trigonometry [14], we can numerically compute the solid angle constituted by arbitrary three vectors $\boldsymbol{r}_{1}, \boldsymbol{r}_{2}$ and $\boldsymbol{r}_{3}$ in the following fashion [15]. First we compute three cosines in terms of inner products of the three vectors as follows:

$$
\begin{aligned}
& \cos \theta_{i}=\frac{\left(\boldsymbol{r}_{\boldsymbol{j}}-w_{i j} \boldsymbol{r}_{\boldsymbol{i}}\right) \cdot\left(\boldsymbol{r}_{\boldsymbol{k}}-w_{i k} \boldsymbol{r}_{\boldsymbol{i}}\right)}{\left|\boldsymbol{r}_{\boldsymbol{j}}-w_{i j} \boldsymbol{r}_{\boldsymbol{i}}\right| \cdot\left|\boldsymbol{r}_{\boldsymbol{k}}-w_{i k} \boldsymbol{r}_{\boldsymbol{i}}\right|} \\
& i, j, k=\{1,2,3\}, \quad i \neq j, k, \quad j \neq k
\end{aligned}
$$

where the weights are computed by the following inner products defined by

$$
\begin{aligned}
& w_{i j}=\frac{\left(\boldsymbol{r}_{\boldsymbol{i}} \cdot \boldsymbol{r}_{\boldsymbol{j}}\right)}{\left(\boldsymbol{r}_{\boldsymbol{i}} \cdot \boldsymbol{r}_{\boldsymbol{i}}\right)}, \quad w_{i k}=\frac{\left(\boldsymbol{r}_{\boldsymbol{i}} \cdot \boldsymbol{r}_{\boldsymbol{k}}\right)}{\left(\boldsymbol{r}_{\boldsymbol{i}} \cdot \boldsymbol{r}_{\boldsymbol{i}}\right)} \\
& i, j, k=\{1,2,3\}, \quad i \neq j, k, \quad j \neq k .
\end{aligned}
$$

Then the required solid angle $\Omega$ in steradian is computed by taking the inverse cosines in Eq.(34) as follows:

$$
\Omega=\theta_{1}+\theta_{2}+\theta_{3}-\pi
$$

Thus we can numerically estimate the solid angle spanned by an arbitrary 3D surface by discretizing it in terms of triangular cells. 


\section{References}

[1] K. Uchida and J. Honda, Estimation of Propagation Characteristics along Random Rough Surface for Sensor Networks, Wireless Sensor Networks: Application-Centric Design, Geoff V Merret and Yen Kheng Tan (Ed.), InTech, Chapter 13, (2010-12) 231-248.

[2] K. Uchida, J. Honda, T. Tamaki and M. Takematsu, Two-Rays Model and Propagation Characteristics in View of Hata's Empirical Equations, IEICE Technical Report, AP2011-14 (2011) 49-54.

[3] K. Uchida, J. Honda and Jun-Hyuck Lee, A Study of Propagation Characteristics and Allocation of Mobile Stations, IEICE Technical Report, IN2011-98, MoMuC2011-32 (2011) pp. 31-36.

[4] M. Hata, Empirical Formula for Propagation Loss in Land Mobile Radio Services, IEEE Trans Veh Technol VT-29 (3) (1980) 317-325.

[5] K. Uchida, M. Takematsu and J. Honda, An Algorithm to Estimate Propagation Parameters Based on 2-Ray Model, Proceedings of NBiS-2012, The 15th International Conference on Network-Based Information Systems, Melbourne, Australia, (2012), 556-561.

[6] K. Uchida, M. Takematsu, J.H. Lee and J. Honda, Field Distributions of 1-Ray Model Using Estimated Propagation Parameters in Comparison with DRTM, Proceedings of BWCCA-2012, 2012 Seventh International Conference on Broadband, Wireless Computing, Communication and Applications, Victoria, Canada, (2012), 488-493.

[7] K. Uchida, K. Shigetomi, M. Takematsu and J. Honda, An Estimation Method for Amplitude Modification Factor Using Floor Area Ratio in Urban Areas, Information Technology Convergence: Security, Robotics, Automations and Communication - Lecture Notes in Electrical Engineering 253, Springer, ISBN 978-94-007-6995-3, (2013), 101-109.

[8] K. Uchida, J. Honda and K.Y. Yoon, Distance Characteristics of Propagation in Relation to Inhomogeneity of Random Rough Surface, Proceedings of ISMOT 2009, The 12th International Symposium on Microwave and Optical Technology, New Delhi, India, (2009), 1133-1136.

[9] J. Honda, K. Uchida and K.Y. Yoon, Estimation of Radio Communication Distance along Random Rough Surface, IEICE Trans. ELECTRON E93-C(1) (2010), 39-45.

[10] K. Uchida and J. Honda, An Algorithm for Allocation of Base Stations in Inhomogeneous Cellular Environment, Proceedings of NBiS-2011, The 14th International Conference on Network-Based Information Systems, Tirana, Albania, (2011), 507-512.

[11] K. Uchida, J. Honda and K.Y. Yoon, An Algorithm for Rough Surface Generation with Inhomogeneous Parameters, Journal of Algorithms and Computational Technology 5 (2) (2011), 259-271.

[12] Y. Mushiake, Antennas and Radio Propagation, Corona Publishing Co., LTD. Tokyo, 1985.

[13] R.E. Collin, Antennas and Radiowave Propagation, McGraw-Hill Book Company, New York, 1985.

[14] G.A. Korn and T.M. Korn, Mathematical Handbook for Scientists and Engineers, McGraw-Hill Book Company (1968), 888-890.

[15] A. Van Oosterom and J. Strackee, The Solid Angle of a Plane Triangle, IEEE Transactions on Biomedical Engineering, BME-30 (2) (1983), 125-126. 

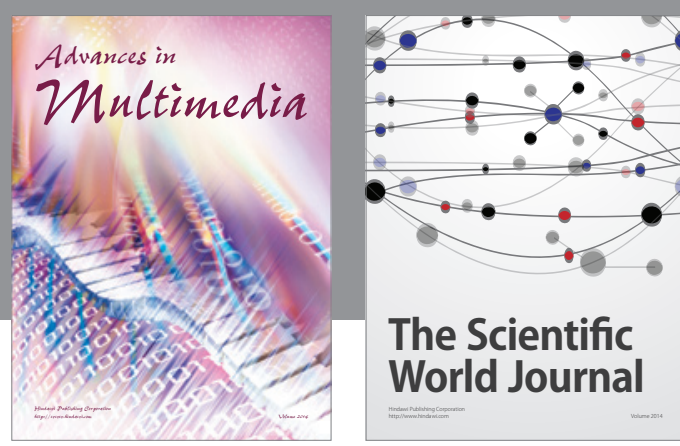

The Scientific World Journal
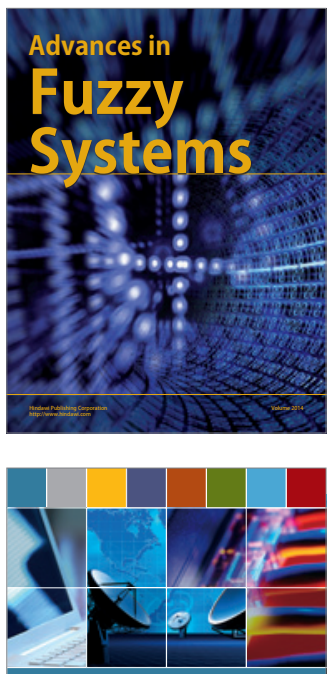

Computer Networks and Communications
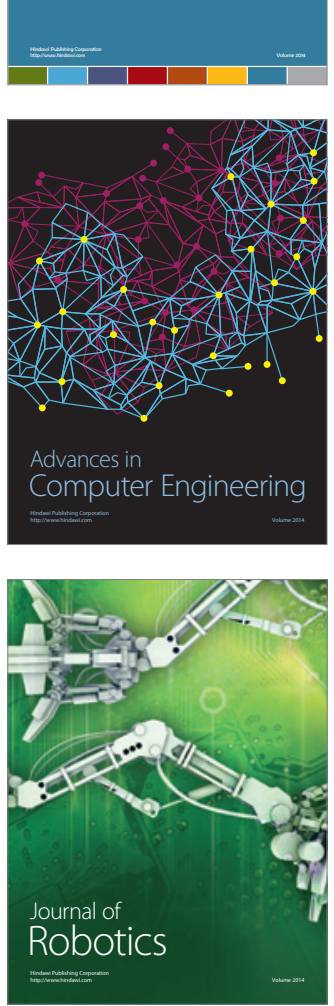
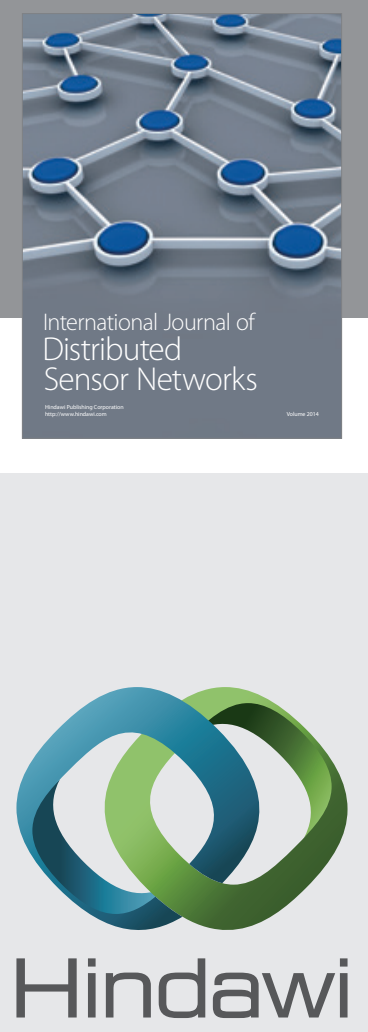

Submit your manuscripts at

http://www.hindawi.com
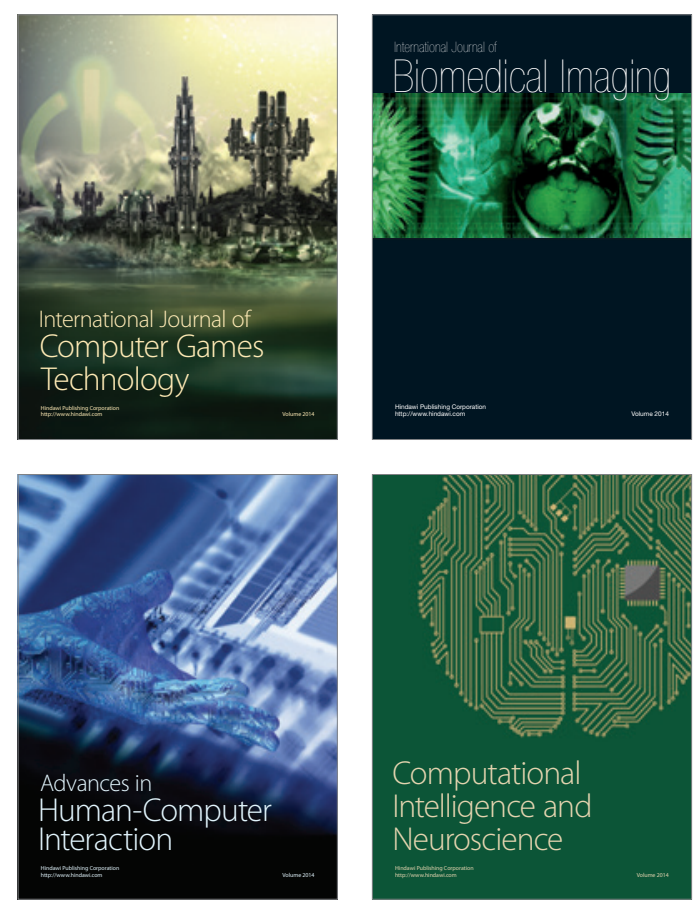
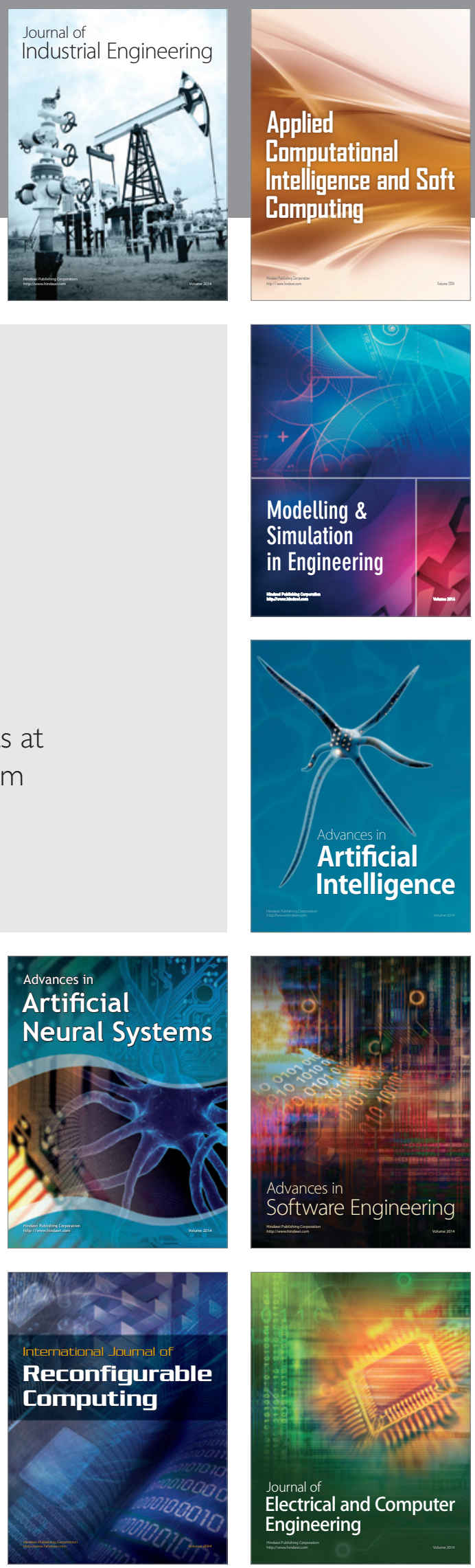